Relations industrielles

Industrial Relations

\title{
Livres reçus Books received
}

Volume 16, numéro 2, avril 1961

URI : https://id.erudit.org/iderudit/1021824ar

DOI : https://doi.org/10.7202/1021824ar

Aller au sommaire du numéro

Éditeur(s)

Département des relations industrielles de l’Université Laval

ISSN

0034-379X (imprimé)

1703-8138 (numérique)

Découvrir la revue

Citer ce document

(1961). Livres reçus. Relations industrielles / Industrial Relations, 16(2), 284-285.

https://doi.org/10.7202/1021824ar

Tous droits réservés @ Département des relations industrielles de l’Université Laval, 1961
Ce document est protégé par la loi sur le droit d'auteur. L'utilisation des services d'Érudit (y compris la reproduction) est assujettie à sa politique d'utilisation que vous pouvez consulter en ligne.

https://apropos.erudit.org/fr/usagers/politique-dutilisation/ 
- Le recrutement et la formation des fonctionnaires: problèmes contemporains », par A. Molitor, Les Dossiers de l'Action Sociale Catholique, 127, rue de la Loi, Bruxelles, 38e année, no 1, janvier 1961, pp. 1-16.

Working Conditions in Canadian Industry, Economics and Research Branch, Department of Labour, Ottawa, 1960, $162 \mathrm{pp}$.

- Les conditions de travail dans l'industrie du cuir et de la chaussure $\gg$, Revue internationale du Travail, Genève, Suisse, vol. LXXXIII, no 2, février 1961, pp. 197-221.

- Importance du personnel âgé de moins de 18 ans employé dans les établissements industriels et commerciaux 》, Reuse française $d u$ Travail, Ministère du Travail, 1, Place de Fontenoy, Paris VIIe, juillet-septembre 1960 , pp. 113116.

« Controlling Unemployment at the Company Level », Industrial Relations Digest, Callaghan \& Company, 165 North Archer Avenue, Mundelein, III., Vol. III, No. 3, January 1961, pp. 39-54.

\section{LIVRES RECUS}

CROWLEY, Desmond, The Background to Current Affairs, The MacMillan Company of Canada Limited, 70 Bond St., Toronto and St. Martin's Press, New York, Second Edition, 1960, 275 pp., $\$ 3.60$.

FRIEDLANDER, Walter A., Introduction to Social Welfare, Prentice-Hall, Inc., Englewood Cliffs, N.J., Second Edition, 1961, 589 pp., \$7.50.

COLE, Arthur H., Business Enterprise in its Social Setting, Harvard University Press, Cambridge, Massachusetts, 1959, 285 pp., \$6.50.

Rapport d'une mission du Bureau international du Travail, Genève, 1960 . La situation syndicale en U.R.S.S. 152 pp., \$1.25.
* Les femmes et l'emploi à temps partiel aux Etats-Unis 》, Revue internationale du Travail, Genève, Suisse, vol. LXXXIII, no 1 , janvier 1961, pp. 97102.

* Work Experience of the (U.S.) Population in 1959 », by Sophia Cooper, Monthly Labor Review, Vol. 83, No. 12,

December 1960, pp. 1272-1283.

* Pay Levels for Professional and Other White-Collar Occupations », Monthly Labor Review, Vol. 83, No. 12, December 1960, pp. 1284-1292.

« White-Collar Employment: I-Trends and Structure, and II-Personal Characteristics, Extent of Employment, Education, and Income », Monthly Labor Review, Vol. 84, Nos. 1 and 2, January and February 1961.

- A Comparative Analysis of the Industrial Structure of the Urban Labor Force of Burma and the United States », by Surinder K. Mehta, Economic Development and Cultural Change, Vol. IX, No. 2, January 1961, pp. 164-179.

Bureau international du Travail, Genève, 1960. La place et le rôle de la direction du personnel dans l'entreprise, série Relations professionnelles: no 7 , documents d'une réunion technique, $242 \mathrm{pp}$.

Bureau international du Travail, Genève, 1960. Le statut et le rôle des représentants des travailleurs, série Relations professionnelles: no 8, documents d'une réunion technique, 161 pp.

International Labour Office, Geneva, 1960. Report to the Government of India on Labour-Management Relations and Some Aspects of Wages Policy, Labour-Management Relations Series: No. 9, 104 pp. 
JANOWITZ, Morris, The Professional Soldier, The Free Press of Glencoe, 119 W. Lake St., Chicago 1, III., 1960, 464 pp. $\$ 6.75$.

RAINWATER, Lee, COLEMAN, Richard P. \& HANDEL, Gerald, Workingman's Wife, Oceana Publications, Inc., 80 Fourth Avenue, New York 3, N.Y., 1959, 238 pp. $\$ 7.50$.

BOWEN, William G., Wage Behavior in the Postwwar Period, An Empirical Analysis, Industrial Relations Section, Princeton University, Princeton, N.J., 1960, $137 \mathrm{pp}$.

CARTWRIGHT, Dorwin, Studies in Social Power, Institute for Social Research, The University of Michigan, Ann Arbor, Michigan, 1959, 225 pp.

Division de la Recherche et de la Statistique, Ministère de la Santé nationale et du Bien-être social, Ottawa. Utilisation de l'hôpital au Canada. Evolution et changements, série des soins de santé, mémoire no 12 , avril 1960 , 1 vol.

Bureau international du Travail, Genève, 1961. Au service de la petite industrie, 245 pp., $\$ 2.00$.

Institut de droit comparé de l'Université de Paris. Les nationalisations en France et d̀ l'étranger - Les nationalisations à l'étranger - Sirey, 22 rue Soufflot, Paris Ve, 1958, 350 pp.

Institut de droit comparé de l'Université de Paris. Etudes de droit contemporain. Contributions françaises aux IIIe et IVe Congrès internationaux de Droit Comparé, I, Sirey, 22, rue Soufflot, Paris Ve, 1959, 304 pp.

Institut de droit comparé de l'Université de Paris. Etudes en droit contemporain. Contributions françaises aux IIIe et IVe Congrès internationaux de Droit Comparé, II, Sirey, 22 rue Soufflot, Paris Ve, 1959, 428 pp.

Institut de droit comparé de l'Université de Paris. Etudes de droit contemporain. Contributions françaises aux
IIIe et IVe Congrès internationaux de Droit Comparé, III, Sirey, 22 rue Soufflot, Paris Ve, 1959, 284 pp.

Institut de droit comparé de l'Université de Paris. Etudes de droit contemporain. Contributions françaises aux IIIe et IVe Congrès internationaux de Droit Comparé, IV, Sirey, 22 rue Soufflot, Paris Ve, 1959, 396 pp.

GALDSTON, Iago, M.D., Editor, Medecine and Anthropology, Lectures to the Laity, No. XXI, International Universities Press, Inc., 227 West 13 Street, New York 11, N.Y., 1959, 165 pp., $\$ 3.00$.

BONNER, HILL \& WILBER, The Contemporary World: The Social Sciences in Historical Perspective, Prentice-Hall, Inc., Englewood Cliffs, N.J., 1960, 594 pp., \$7.95.

KNOWLES, William H., Trade Union Development \& Industrial Relations in the British West Indies. University of California Press, Berkeley, California, 1959, 214 pp., \$5.00.

PHELPS, Orme W., Discipline \& Discharge in the Unionized Firm. University of California Press, Berkeley, California, 1959, 149 pp.

MEYERS, Frederic, European Coal Mining Unions: Structure and Functions, Institute of Industrial Relations, University of California, Los Angeles, 1961,161 pp., $\$ 2.75$.

SPYROPOULOS, Georges, L'administration des oeuvres sociales par les comités d'entreprise, R. Pichon \& R. Durand-Auzias, 20, rue Soufflot, Paris Ve, 1961, 142 pp., 16, 70 NF.

GALENSON, Walter, Trade Union Democracy in Western Europe. Institute of Industrial Relations, University of California, University of California Press, Berkeley and Los Angeles, 1961, 97 pp. p. $\$ 2.25$. 